\section{TNF in hot shocker}

\begin{abstract}
Sitting in direct sunlight should usually be avoided, but now it seems that there might be some benefit, at least, to getting hot. According to new research in Immunity, 'heat shocking' protects against the destructive effects of tumour-necrosis factor (TNF) in mice. Scientists from Belgium and Japan have shown that heat shock protects against the lethal effects of $\mathrm{TNF}$ in mice and can reduce the death rate during TNF antitumour therapy, without affecting the treatment efficacy.

Heat-shock proteins (HSPs) are a group of conserved molecules that are present in all organisms. Under normal physiological conditions, the cellular expression of these proteins is low. However, stress stimuli, including environmental factors or pathological events, cause a great increase in their expression, particularly HSP70. TNF is a cytokine produced by macrophages that has strong antitumour activity both in vitro and in vivo. However, high doses of systemic TNF induce systemic inflammatory-response syndrome - characterized by bowel
\end{abstract}
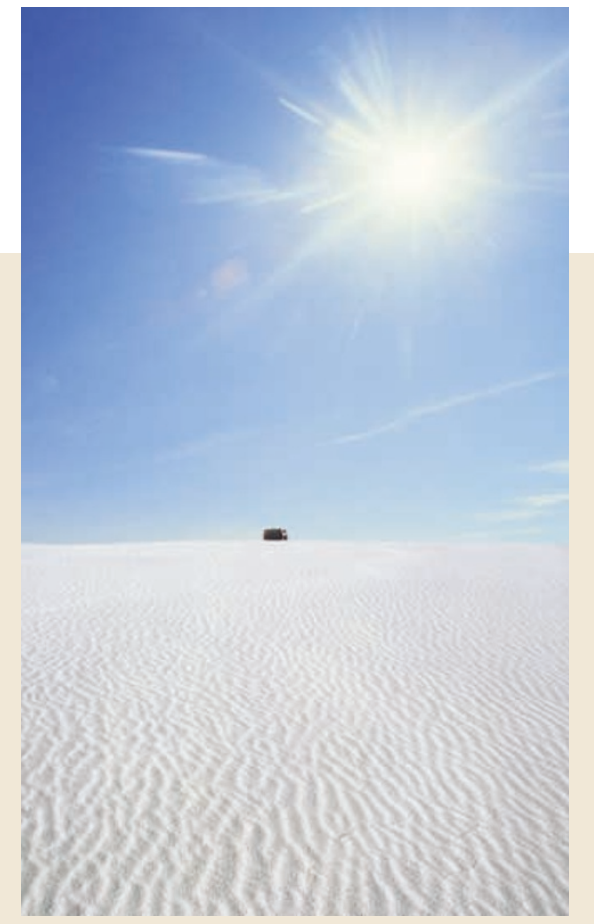

necrosis, liver damage, severe hypotension and death — which limits the effectiveness of TNF as a systemic anticancer treatment.

To heat shock mice, the body temperature of the mice was elevated from $37^{\circ} \mathrm{C}$ to $41.5^{\circ} \mathrm{C}$ over a period of 20 minutes, which caused an increase in HSP70 production in various organs between 6 to 24 hours later. These mice were protected from TNF-induced death when treated 12 hours after heat shock. A lethal dose of TNF resulted in the production of large amounts of the inflammatory cytokine interleukin-6 (IL-6) and nitric oxide (NO), which caused hypotension. Applying a heat shock 12 to 24 hours before the TNF challenge resulted in a statistically significant reduction in the production of NO and IL-6. This treatment also completely prevented apoptosis in the gut. To confirm whether the protective effects of heat shock were mediated by HSP70, the experiments were repeated in Hsp70-deficient mice. None of the protection from heat shock was seen in the Hsp70-deficient mice compared with wild-type mice after challenge with lethal doses of TNF.

Libert and colleagues tested whether heat shock would reduce some of the toxic effects of TNF as a cancer treatment, while retaining its efficacy. In a mouse melanoma model in which treatment with TNF in combination with interferon- $\gamma($ IFN- $\gamma)$ was effective in causing tumour regression but was accompanied by high mortality, heat-shock treatment reduced toxicity while retaining efficacy. At present, TNF is used in combination with IFN $-\gamma$ and melphalan to treat tumours in the extremities in a process known as isolated limb perfusion; however, local toxicity can be a problem. Heat shock could be a simple therapeutic treatment to inhibit TNF toxicity in perfusion treatments, as well as other TNF-related syndromes.

\section{Melanie Brazil}

(2) References and links

ORIGINAL RESEARCH PAPER Van Molle, W. et al. HSP70 protects against TNF-induced lethal inflammatory shock. Immunity 16, 685-695 (2002) WEB SITES

Libert's laboratory: http://www.dmb.rug.ac.be

\section{ANTICANCER DRUGS}

\section{Deadly cargo}

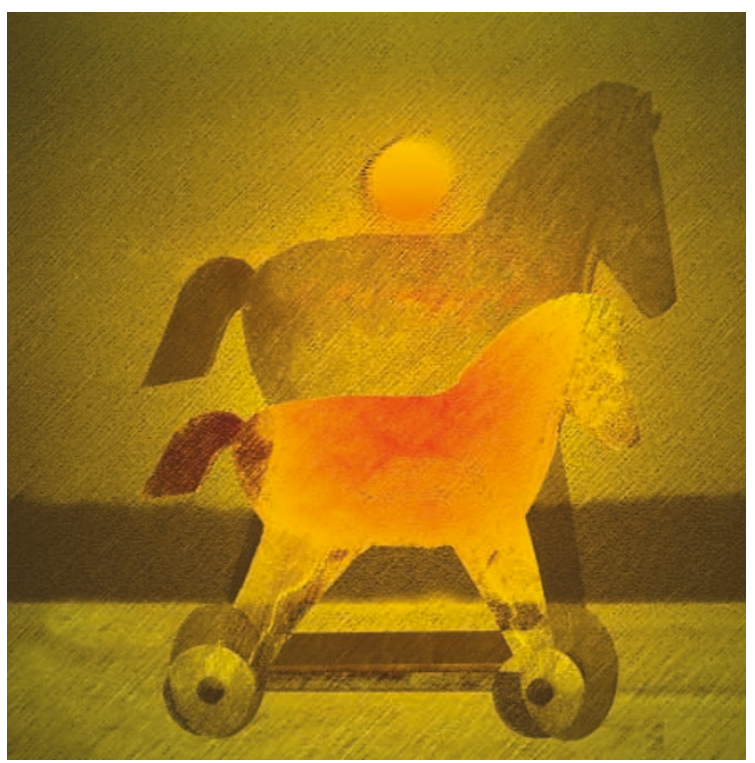

Angiogenesis - the formation of new blood vessels - is essential for tumour progression and metastasis. Vascular endothelial growth factor (VEGF) has a key role in this process that seems to be mediated mainly by one of its receptors, $\mathrm{KDR}$, which is overexpressed on the tumour vasculature. Writing in Proceedings of the National Academy of Sciences, Veenendaal et al. describe how this could be therapeutically exploited by fusing the toxin gelonin ( $\mathrm{rGel}$ ) to VEGF to selectively destroy the tumour vasculature in vivo.

The fusion protein, which consists of VEGF (one of at least four human VEGF isoforms) linked by a flexible tether to rGel, was expressed in Escherichia coli and purified, and shown to activate KDR in the same manner as VEGF alone. Gelonin was chosen as the toxin as it does not seem to be antigenic in humans, and unlike other toxins assessed so far for antitumour therapies - it does not seem to cause damage to normal blood vessels.

In in vitro tests, $\mathrm{VEGF}_{121} / \mathrm{rGel}$ was highly toxic $\left(\mathrm{IC}_{50} \leq 1 \mathrm{~nm}\right)$ to dividing endothelial cells that overexpressed the KDR receptor, but cells expressing lower levels of KDR were no more sensitive to $\mathrm{VEGF}_{121} / \mathrm{rGel}$ than to free $\mathrm{rGel}\left(\mathrm{IC}_{50} \geq\right.$
$300 \mathrm{~nm}$ ). This requirement to surpass a threshold level of KDR expression could be important for the safety of $\mathrm{VEGF}_{121} / \mathrm{rGel}$, as the level of KDR expression on normal organs is likely to be below the threshold. Moreover, $\mathrm{VEGF}_{121} / \mathrm{rGel}$ was 60 -fold more toxic to dividing cells than nondividing cells expressing the same levels of KDR.

The promise of the in vitro results was well borne out in vivo - in mice with humanmelanoma or human-prostate xenografts, treatment with $\mathrm{VEGF}_{121} / \mathrm{rGel}$ resulted in a reduction in tumour volume to $<17 \%$ of the untreated controls. $\mathrm{VEGF}_{121} / \mathrm{rGel}$ was localized primarily on the vascular endothelium of the tumours, and vascular damage and thrombosis of tumour blood vessels was observed, in contrast to normal tissues, in which the vasculature seemed unaffected. Overall, it seems that selective destruction of the tumour vasculature can be achieved with $\mathrm{VEGF}_{121} / \mathrm{rGel}$ in mice, and human trials are expected to begin in the next year.

Peter Kirkpatrick

(9) References and links

ORIGINAL RESEARCH PAPER Veenendaal, L. M. et al. In vitro and in vivo studies of a VEGF/rGelonin chimeric fusion toxin targeting the neovasculure of sold th . Natl Acad. Sci. USA 99, 7866-7871 (2002) 